\title{
Log-transformed plasma level of brain natriuretic peptide during the acute phase of Kawasaki disease is quantitatively associated with myocardial dysfunction
}

Sunhee Bang, $M D^{1}$, Jeong Jin $Y u, M D^{1}$, MyungKi Han, MD', Hong Ki Ko, MD', Sail Chun, $\mathrm{MD}^{2}$, Hyung Soon Choi, MD, Young-Hwue Kim, MD', Jae-Kon Ko, MD', In-Sook Park, $\mathrm{MD}^{1}$

${ }^{1}$ Division of Pediatric Cardiology, Departments of Pediatrics and ${ }^{2}$ Laboratory Medicine, University of Ulsan College of Medicine, Seoul, ${ }^{3}$ Department of Pediatrics, Kosin University College of Medicine, Busan, Korea
Purpose: Brain natriuretic peptide (BNP) has been considered a biochemical marker for myocarditis in Kawasaki disease. We performed this study to determine its quantitative significance.

Methods: We attempted to correlate log-transformed BNP concentrations (log-BNP) and clinical, laboratory, and echocardiographic variables in 81 children with Kawasaki disease. Stepwise multiple linear regression analysis was used to determine the variables independently associated with log-BNP concentration.

Results: Serum C-reactive protein level $(P<0.0001)$, serum alanine aminotransferase concentration $(P=0.0032)$, white blood cell count $(P=0.0030)$, and left ventricular mass index $(P=0.0024)$ were positively related with log-BNP, and hemoglobin level $(P<0.0001)$, serum albumin level $(P<0.0001), \mathrm{Na}^{+}$concentrations $(P<0.0001)$, left ventricular fractional shortening ( $P=0.0080)$, and peak early diastolic tissue velocity of the left ventricular basal lateral segment $(P=0.0045)$ were negatively related to the log-BNP concentration. Multiple regression analysis showed that serum albumin concentration $\left(R_{2}=0.31\right.$, $P=0.0098)$ and left ventricular mass index $\left(R_{2}=0.09, P=0.0004\right)$ were significantly associated with the log-BNP concentration.

Conclusion: Elevated BNP levels during the acute phase of Kawasaki disease may be attributable to cardiac dysfunction associated with the increase in left ventricular mass, and log-BNP concentration may be a quantitative biochemical marker of myocarditis in Kawasaki disease.

Key words: Kawasaki disease, Myocarditis, Brain natriuretic peptide
Received: 13 December 2010, Revised: 3 May, 2011

Accepted: 21 July 2011

Corresponding author: Jeong Jin Yu, MD

Division of Pediatric Cardiology, Department of Pediatrics, Asan Medical Center, University of Ulsan College of Medicine, 388-1 Pungnap-dong, Songpa-gu, Seoul 138-736, Korea

Tel: +82-2-3010-3924, Fax: +82-2-473-3725

E-mail: pediatrist@medimail.co.kr

Copyright $(02011$ by The Korean Pediatric Society

This is an open-access article distributed under the terms of the Creative Commons Attribution Non-Commercial License (http://creativecommons.org/licenses/by-
nc/3.0/) which permits unrestricted non-commercial use, distribution, and reproduction in any medium, provided the original work is properly cited.

\section{Introduction}

Brain natriuretic peptide (BNP) has been considered a biochemical marker for myocarditis during the acute phase of Kawasaki disease ${ }^{1-4)}$.
Plasma BNP levels increased more than $16.8 \mathrm{pg} / \mathrm{mL}$ during the acute phase in $87.5 \%$ of patients $^{2}$, and decreased to nearly normal levels during the convalescent phase in most of the patients ${ }^{1,2}$. Although increased plasma concentrations of BNP $(>50 \mathrm{pg} / \mathrm{mL})$ have been 
regarded as an indicator of myocarditis in Kawasaki disease during the acute phase ${ }^{1,4)}$, the association between BNP quantitation and other parameters, including cardiac systolic dysfunction and coronary arterial dilatation, has been in controversy ${ }^{1,4-6)}$. A recent study reported a significant relation between diastolic function with BNP, but data of diastolic function in that study did not differ between patients and controls ${ }^{3)}$.

We have therefore sought to determine the clinical, laboratory and echocardiographic variables independently associated with quantitative variations in BNP in a relatively large number of patients with Kawasaki disease.

\section{Methods}

The study subjects consisted of 81 children registered at the Asan Medical Center from March 2009 through March 2010 who fulfilled the diagnostic criteria for Kawasaki disease ${ }^{7)}$. All patients were in acute phase and underwent echocardiographic examination before intravenous immunoglobulin infusion therapy. One patient had a familial history of Kawasaki disease. Dilatation of coronary arteries according to criteria of Japanese Ministry of Health and Welfare $^{8)}$ was found in 4 patients, one among them had aneurysmal dilatation. Of the 81 patients, 20 were enrolled in another study on myocardial deformation during the acute phase of Kawasaki disease?

Demographic and laboratory data were surveyed by reviewing medical records. Plasma BNP concentration was determined by a chemiluminescent immunoassay (Siemens Healthcare Diagnostics, Deerfield, IL, USA), and was log transformed (log-BNP). Because BNP concentrations $>50 \mathrm{pg} / \mathrm{mL}$ during the acute phase of Kawasaki disease have been reported to be an qualitative indicator of myocarditis ${ }^{1,4)}$, patients were divided into 2 groups based on their BNP concentrations, with group 1 consisting of 37 patients with $\mathrm{BNP}>50 \mathrm{pg} / \mathrm{mL}$ and group 2 consisting of 44 patients with BNP $\leq 50 \mathrm{pg} / \mathrm{mL}$.

The study protocol was approved by the Institutional Review Board, Asan Medical Center, Seoul, Korea. All subjects or their parents provided informed consent.

\section{Echocardiographic examinations}

Subjects were prepared for echocardiography by administering a sedative and/or antipyretic, as required. All echocardiographic examinations were performed by a single echocardiographer, and images were obtained using a Vivid 7 Vantage machine equipped with 7-MHz and 5-MHz transducers (GE Vingmed, Horten, Norway). All recordings of 2D, M-mode, and Doppler images were obtained according to the recommendations of the American Society of Echocardiography ${ }^{10-12)}$. Moving images (40 to 100 frames/sec) were stored for analysis of tissue velocity from apical four-chamber view, and measurements performed off-line using EchoPAC PC ver. 7 (GE Vingmed, Horten Norway) by another author blinded to patient status. Left ventricular fractional shortening was calculated from dimensions measured from the standard M-mode using the parasternal long axis view. Left ventricular mass was calculated using the formula derived by Devereux and Reichek ${ }^{13)}$, and the index was obtained by dividing the mass by body surface area.

Tissue velocities of the basal lateral segment of the left ventricle were determined by analysis of 2D strain using speckle tracking imaging. Data were approved when considered acceptable by the software. In 7 patients, tissue velocity data were abandoned owing to failure to meet approval. We also calculated the ratio of peak early diastolic velocity of mitral inflow (E) to peak early diastolic tissue velocity of the lateral basal segment (Em).

Fifteen moving images were randomly selected, and tissue velocities of the basal lateral segment of the left ventricle (peak systolic tissue velocity [Sm], Em, and peak late diastolic tissue velocity [Am]) were determined by 1 observer on 2 occasions and by 2 observers on 2 occasions. Reproducibility of measurement of tissue velocities was tested though Pearson correlation analysis. Results of intraobserver test were $P=0.0010 / \mathrm{R}=0.78$ in $\mathrm{Sm}, P<0.0001 / \mathrm{R}=0.84$ in $\mathrm{Em}$, and $P<0.0001 / \mathrm{R}=0.96$ in Am. Results of interobserver test were $P=0.0242 / \mathrm{R}=0.60$ in $\mathrm{Sm}, P=0.0019 / \mathrm{R}=0.75$ in $\mathrm{Em}$, and $P=0.1559$ / $\mathrm{R}=0.40$ in $\mathrm{Am}$.

\section{Statistical analysis}

All data are presented as means \pm standard deviations (SDs). All statistical analyses were performed using SAS ver. 9.1 (SAS Institute Inc., Cary, NC, USA), and statistical significance was defined as a $P$ value $<0.01$. The skewness of distribution of BNP / log-BNP was calculated using the formula:

Skewness $=3 \times($ mean-median $) / S D$

Chi-squared analysis was used to determine whether the distribution of categorical variables differed between groups. Student's t-tests were used to compare numerical variables between groups. Correlations between the BNP / log-BNP and clinical, laboratory and echocardiographic variables were assessed using Pearson correlation analysis. Stepwise multiple linear regression analysis (SLS 0.15, SLE 0.15 ) was used to determine the variables significantly associated with $\log$-BNP.

\section{Results}

The demographic and anthropometric data did not differ signi- 
Table 1. Between-Group Comparisons of Clinical and Laboratory Data

\begin{tabular}{|c|c|c|c|}
\hline Variable & Group 1* $(n=37)$ & Group $2^{\dagger}(n=44)$ & $P$ value \\
\hline Males & $19(51.4)$ & $20(45.4)$ & 0.5967 \\
\hline Age (yr) & $2.16 \pm 1.80$ & $2.41 \pm 1.84$ & 0.5433 \\
\hline Body weight (kg) & $11.7 \pm 4.1$ & $13.2 \pm 5.0$ & 0.1448 \\
\hline Height (cm) & $84.5 \pm 14.3$ & $89.3 \pm 16.4$ & 0.1799 \\
\hline Duration of fever (day) & $6.4 \pm 1.7$ & $6.1 \pm 1.5$ & 0.4046 \\
\hline $\mathrm{BNP}(\mathrm{pg} / \mathrm{mL})$ & $273.7 \pm 391.2$ & $21.1 \pm 13.3$ & $<0.0001$ \\
\hline Log-BNP & $2.23 \pm 0.37$ & $1.21 \pm 0.36$ & $<0.0001$ \\
\hline $\mathrm{CRP}(\mathrm{mg} / \mathrm{dL})$ & $14.1 \pm 8.1$ & $7.8 \pm 7.4$ & 0.0004 \\
\hline WBC $\left(\times 10^{3} / \mathrm{mm}^{3}\right)$ & $15.9 \pm 4.4$ & $13.4 \pm 4.2$ & 0.0104 \\
\hline Hemoglobin (g/dL) & $10.66 \pm 1.04$ & $11.35 \pm 0.99$ & 0.0032 \\
\hline Platelets $\left(x 10^{3} / \mathrm{mm}^{3}\right)$ & $325.9 \pm 68.1$ & $335.9 \pm 93.9$ & 0.7851 \\
\hline Albumin (g/dL) & $3.01 \pm 0.60$ & $3.55 \pm 0.50$ & $<0.0001$ \\
\hline sALT (U/L) & $150.0 \pm 133.6$ & $85.5 \pm 133.2$ & 0.0332 \\
\hline $\mathrm{Na}^{+}(\mathrm{mEq} / \mathrm{L})$ & $134.2 \pm 2.9$ & $136.2 \pm 2.3$ & 0.0007 \\
\hline Pyuria & $10(27.0 \%)$ & $10(22.7 \%)$ & 0.6549 \\
\hline
\end{tabular}

Values are presented as number (\%) or mean \pm SD.

BNP, brain natriuretic peptide; Log-BNP, log transformed BNP; CRP, C-reactive protein; WBC, white blood cell count; SALT, serum alanine aminotransferase.

*Group 1, BNP >50 pg/mL. 'Group 2, BNP $\leq 50$ pg/mL.
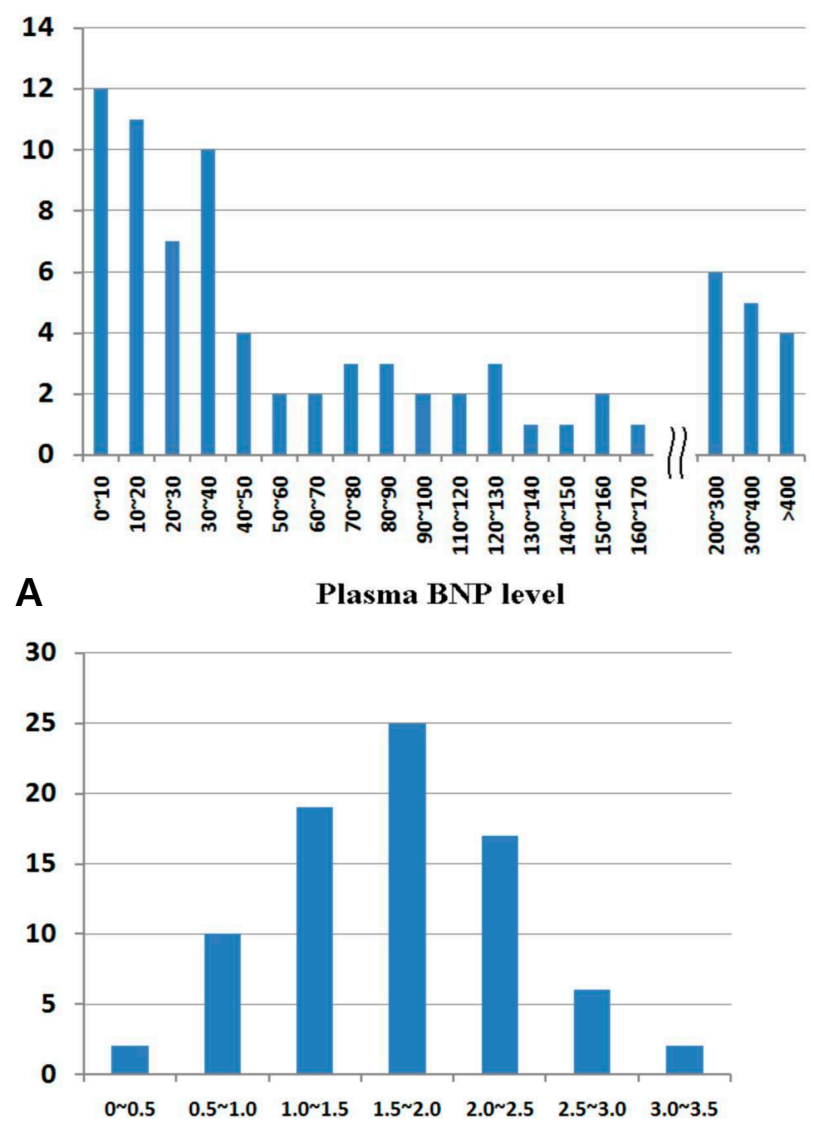

B

$\log -\mathbf{B N P}$

Fig. 1. Distribution of (A) brain natriuretic peptide (BNP) and (B) logtransformed BNP concentration (log-BNP) in 81 patients with Kawasaki disease. ficantly among groups (Table 1). The distributions of BNP and logBNP are shown in Fig. 1. The mean $\pm S D$, median, and skewness of BNP were $136.5 \pm 291.5 \mathrm{pg} / \mathrm{mL}, 40.7 \mathrm{pg} / \mathrm{mL}$, and 0.99 , respectively, whereas them of $\log$-BNP were $1.68 \pm 0.63,1.61$, and 0.31 , respectively.

Serum C-reactive protein (CRP) concentration was significantly higher $(P=0.0004)$, whereas hemoglobin $(P=0.0032)$, serum albumin $(P<0.0001)$ and $\mathrm{Na}^{+}(P=0.0007)$ concentrations were significantly lower, in group 1 than in group 2.

Mitral regurgitation and pericardial effusion was detected in 20 and in 10 patients respectively, but severities of them looked to be less than hemodynamic significance and the distribution of them was not different between groups (Table 2). When we compared numerical echocardiographic variables, we found that heart rate $(P=0.0023)$ and E/Em ratio $(P=0.0066)$ were significantly higher, and $\operatorname{Sm}(P=0.0079)$ was significantly lower, in group 1 than in group 2 (Table 2).

The laboratory and echocardiographic variables significantly related to BNP and/or log-BNP are shown in Table 3. White blood cell count, hemoglobin and serum alanine aminotransferase concentrations, left ventricular fractional shortening and Em were

Table 2. Between-Group Comparisons of Echocardiographic Data

\begin{tabular}{|c|c|c|c|}
\hline Variable & Group 1* $(n=37)$ & Group $2^{\dagger}(n=44)$ & $P$ value \\
\hline Heart rate (bpm) & $136.9 \pm 17.3$ & $124.9 \pm 17.1$ & 0.0023 \\
\hline Mitral regurgitation & $14(37.8)$ & $6(13.6)$ & 0.0566 \\
\hline Pericardial effusion & $6(16.2)$ & $4(9.0)$ & 0.3315 \\
\hline LMCA (mm) & $2.26 \pm 0.42$ & $2.24 \pm 0.51$ & 0.8612 \\
\hline $\mathrm{LAD}(\mathrm{mm})$ & $1.92 \pm 0.42$ & $1.88 \pm 0.52$ & 0.6915 \\
\hline $\mathrm{RCA}(\mathrm{mm})$ & $1.86 \pm 0.49$ & $1.76 \pm 0.66$ & 0.4816 \\
\hline LVDd (mm) & $30.1 \pm 4.5$ & $31.1 \pm 4.4$ & 0.3026 \\
\hline LVDs (mm) & $20.5 \pm 4.4$ & $20.4 \pm 3.8$ & 0.8675 \\
\hline LVFS (\%) & $32.3 \pm 5.6$ & $34.7 \pm 5.0$ & 0.0477 \\
\hline LV mass index $\left(\mathrm{g} / \mathrm{m}^{2}\right)$ & $75.8 \pm 14.2$ & $69.2 \pm 10.0$ & 0.0172 \\
\hline $\mathrm{E}(\mathrm{m} / \mathrm{sec})$ & $1.18 \pm 0.20$ & $1.09 \pm 0.16$ & 0.0391 \\
\hline $\mathrm{A}(\mathrm{m} / \mathrm{sec})$ & $0.95 \pm 0.26$ & $0.87 \pm 0.22$ & 0.1586 \\
\hline$E / A$ & $1.31 \pm 0.34$ & $1.31 \pm 0.30$ & 0.9704 \\
\hline $\mathrm{Sm}(\mathrm{cm} / \mathrm{sec})$ & $4.60 \pm 1.37$ & $5.74 \pm 2.07$ & 0.0079 \\
\hline $\mathrm{Em}(\mathrm{cm} / \mathrm{sec})$ & $7.25 \pm 2.07$ & $8.45 \pm 1.88$ & 0.0110 \\
\hline $\operatorname{Am}(\mathrm{cm} / \mathrm{sec})$ & $5.93 \pm 2.29$ & $6.64 \pm 2.76$ & 0.2368 \\
\hline $\mathrm{E} / \mathrm{Em}$ & $17.4 \pm 6.7$ & $13.6 \pm 4.6$ & 0.0066 \\
\hline
\end{tabular}

Values are presented as mean \pm SD or number (\%).

bpm, beats per minute; LMCA, diameter of left main coronary artery; LAD, diameter of left anterior descending coronary artery; RCA, diameter of right coronary artery; LVDd, left ventricular end-diastolic dimension; LVDs, left ventricular end-systolic dimension; LVFS, left ventricular fractional shortening; E peak, early diastolic velocity of mitral inflow; A peak, atrial diastolic mitral inflow; Sm, systolic tissue velocity; Em, early diastolic tissue velocity; Am, late diastolic tissue velocity.

*Group 1, BNP >50 pg/mL, ${ }^{\dagger}$ Group 2, BNP $\leq 50$ pg/mL. 
Table 3. Correlations between Laboratory and Echocardiographic Variables, and BNP and log-BNP Levels

\begin{tabular}{|c|c|c|c|c|}
\hline & \multicolumn{2}{|c|}{ BNP } & \multicolumn{2}{|c|}{ Log-BNP } \\
\hline & $\mathrm{R}$ & $P$ value & $\mathrm{R}$ & $P$ value \\
\hline CRP & 0.34 & 0.0020 & 0.51 & $<0.0001$ \\
\hline WBC & 0.21 & 0.0641 & 0.33 & 0.0030 \\
\hline Hemoglobin & -0.21 & 0.0648 & -0.44 & $<0.0001$ \\
\hline Albumin & -0.46 & $<0.0001$ & -0.58 & $<0.0001$ \\
\hline sGPT & 0.20 & 0.0677 & 0.32 & 0.0032 \\
\hline $\mathrm{Na}+$ & -0.42 & 0.0001 & -0.44 & $<0.0001$ \\
\hline LVFS & -0.27 & 0.0136 & -0.29 & 0.0080 \\
\hline LV mass index & 0.41 & 0.0001 & 0.33 & 0.0024 \\
\hline Em & 0.10 & 0.4073 & 0.33 & 0.0045 \\
\hline
\end{tabular}

BNP, brain natriuretic peptide; Log-BNP, log transformed BNP; R, Pearson's correlation coefficient; CRP, C-reactive protein; WBC, white blood cell count; sGPT, serum alanine aminotransferase; LVFS, left ventricular fractional shortening; Em, early diastolic tissue velocity.

significantly related to log-BNP, but not to BNP. CRP, serum albumin and $\mathrm{Na}^{+}$concentrations were significantly related to both $\mathrm{BNP}$ and $\log$-BNP, but correlation coefficients were higher for logBNP than for BNP. Heart rate was not significantly related to BNP $(P=0.3267)$ and $\log -\mathrm{BNP}(P=0.0319)$. Diameters of coronary arteries were not related to BNP (left main coronary artery, $P=0.5497$; left anterior descending coronary artery, $P=0.4443$; right coronary artery, $P=0.2351)$ and $\log -\mathrm{BNP}(P=0.5621, P=0.3136, P=0.2259)$.

Significant independent variables resulted from stepwise multiple linear regression analysis, in which log-BNP was the dependent variable, were serum albumin $\left(\mathrm{R}^{2}=0.31, P=0.0098\right)$, left ventricular mass index $\left(\mathrm{R}^{2}=0.09, P=0.0004\right)$, CRP $\left(\mathrm{R}^{2}=0.06, P=0.0073\right)$, fractional shortening $\left(\mathrm{R}^{2}=0.05, P=0.0006\right)$, and end diastolic dimension $\left(\mathrm{R}^{2}=0.05, P<0.0001\right)$.

\section{Discussion}

Limitations of previous studies ${ }^{1-4,6)}$ on the relationship between the myocardial dysfunction and increased BNP levels during the acute phase of Kawasaki disease might be the small numbers of subjects and the rightward skewness of the distribution of BNP. We found, however, that this skewness could be overcome by log transformation of BNP, allowing successful correlation and multiple regression analyses of various clinical factors with $\log$-BNP.

Log-BNP showed significant correlation with laboratory variables that reflecting the severity of disease during the acute phase, and/or that were laboratory diagnostic criteria for incomplete presentation of Kawasaki disease ${ }^{7,14,15)}$, indicating that log-BNP may quantitatively reflect the severity of Kawasaki disease during the acute phase. Moreover, log-BNP may be a better marker of myocarditis in Kaw- asaki disease than of coronary artery complications. We found that the diameters of the coronary arteries were not significantly related to BNP or log-BNP, although several left ventricular systolic and diastolic function indices were significantly related to $\log$-BNP in multiple regression and correlation analyses. Moreover, several systolic and diastolic function variables showed more significantly deviated values in patients with high than with low BNP. The relation between pericardial effusion and elevation of BNP has been in controversy ${ }^{2,16)}$, but we found no significant relationship between them.

We found that serum albumin concentration and left ventricular mass index were significant independent predictors of log-BNP, with relatively higher $\mathrm{R}$ square values than other variables. Increased vascular permeability from vasculitis and resultant vascular leakage are key features of the pathophysiology of Kawasaki disease, and increased vascular leakage may cause hypoalbuminemia and tissue edema ${ }^{15}$. We previously showed that serum albumin concentration and left ventricular mass index were significantly associated during the acute phase of Kawasaki disease, which suggests that increases in left ventricular mass index may result from myocardial swelling caused by increased vascular permeability ${ }^{17}$. Increases in left ventricular mass have been significantly associated with diastolic dysfunctions ${ }^{17}$. Thus, our findings that $\log$-BNP was significantly associated with serum albumin concentration and left ventricular mass index, indicate a causative association between increases in left ventricular mass caused by myocardial swelling and myocardial systolic/diastolic dysfunction that induces secretion of BNP from the myocardium. It is generally well known mechanism of elevation of BNP level, that ventricular production of BNP is enhanced in the presence of left ventricular hypertrophy and in the presence of associated systolic/ diastolic dysfunction ${ }^{18}$. Is this mechanism unchanged in patients with Kawasaki disease during the acute phase? The answer is not confirmative because the elevation of left ventricular mass during the acute phase of Kawasaki disease may not be a compensatory result against an overwelming hemodynamic load. Whatever the mechanism of left ventricular hypertrophy, we speculate that it may be a cause of myocardial dysfunction, which induced the increased secretion of BNP. Already we showed the association between an increased left ventricular mass and the myocardial systolic/diastolic dysfunction in the acute phase of Kawasaki disease ${ }^{9,177}$.

Our study had several limitations. First, the dataset used for analysis was not complete. In seven patients, tissue velocity analyses had to be excluded due to failure of imaging approval. Second, some results of reproducibility tests were unsatisfactory.

In conclusion, the elevation of BNP during the acute phase of Kawasaki disease may be due to cardiac dysfunction associated with the increase in left ventricular mass. Log-BNP was significantly 
related to laboratory variables reflective of disease severity and to systolic/diastolic myocardial dysfunction, suggesting that log-BNP may be a quantitative biochemical marker of myocarditis in Kawasaki disease.

\section{References}

1. Kawamura T, Wago M. Brain natriuretic peptide can be a useful biochemical marker for myocarditis in patients with Kawasaki disease. Cardiol Young 2002;12:153-8.

2. Kawamura T, Wago M, Kawaguchi H, Tahara M, Yuge M. Plasma brain natriuretic peptide concentrations in patients with Kawasaki disease. Pediatr Int 2000;42:241-8.

3. Kurotobi S, Kawakami N, Shimizu K, Aoki H, Nasuno S, Takahashi $\mathrm{K}$, et al. Brain natriuretic peptide as a hormonal marker of ventricular diastolic dysfunction in children with Kawasaki disease. Pediatr Cardiol 2005;26:425-30.

4. Takeuchi D, Saji T, Takatsuki S, Fujiwara M. Abnormal tissue doppler images are associated with elevated plasma brain natriuretic peptide and increased oxidative stress in acute Kawasaki disease. Circ J 2007;71:35762.

5. Iwashima S, Ishikawa T, Ohzeki T. Brain natriuretic peptide levels in Kawasaki disease: a case report. Pediatr Int 2009;51:415-8.

6. Zhang QY, Du JB, Chen YH, Li WZ. Change in plasma N-terminal probrain natriuretic peptide in children with Kawasaki disease and its value in clinical practice. Zhonghua Er Ke Za Zhi 2006;44:886-90.

7. Newburger JW, Takahashi M, Gerber MA, Gewitz MH, Tani LY, Burns JC, et al. Diagnosis, treatment, and long-term management of Kawasaki disease: a statement for health professionals from the Committee on Rheumatic Fever, Endocarditis, and Kawasaki Disease, Council on Cardiovascular Disease in the Young, American Heart Association. Pediatrics 2004;114:1708-33.

8. Research Committee on Kawasaki disease. Report of subcommittee on standardization of diagnostic criteria and reporting of coronary artery lesions in Kawasaki disease. Tokyo (JP): Japaness Ministry of Health and Welfare, 1984.
9. Yu JJ, Choi HS, Kim YB, Son JS, Kim YH, Ko JK, et al. Analyses of left ventricular myocardial deformation by speckle-tracking imaging during the acute phase of Kawasaki disease. Pediatr Cardiol 2010;31:807-12.

10. Quiñones MA, Otto CM, Stoddard M, Waggoner A, Zoghbi WA; Doppler Quantification Task Force of the Nomenclature and Standards Committee of the American Society of Echocardiography. Recommendations for quantification of Doppler echocardiography: a report from the Doppler Quantification Task Force of the Nomenclature and Standards Committee of the American Society of Echocardiography. J Am Soc Echocardiogr 2002;15:167-84.

11. Sahn DJ, DeMaria A, Kisslo J, Weyman A. Recommendations regarding quantitation in M-mode echocardiography: results of a survey of echocardiographic measurements. Circulation 1978;58:1072-83.

12. Schiller NB, Shah PM, Crawford M, DeMaria A, Devereux R, Feigenbaum H, et al. Recommendations for quantitation of the left ventricle by two-dimensional echocardiography. American Society of Echocardiography Committee on Standards, Subcommittee on Quantitation of Two-Dimensional Echocardiograms. J Am Soc Echocardiogr 1989;2:358-67.

13. Devereux RB, Reichek N. Echocardiographic determination of left ventricular mass in man. Anatomic validation of the method. Circulation 1977;55:613-8.

14. Muta H, Ishii M, Egami K, Hayasaka S, Nakamura Y, Yanagawa H, et al. Serum sodium levels in patients with Kawasaki disease. Pediatr Cardiol 2005;26:404-7.

15. Terai M, Honda T, Yasukawa K, Higashi K, Hamada H, Kohno Y. Prognostic impact of vascular leakage in acute Kawasaki disease. Circulation 2003;108:325-30.

16. Kim HJ, Jeong YS, Jung JW. Plasma brain natriuretic peptide concentration and tissue doppler imaging for acute febrile phase in patients with Kawasaki disease. J Korean Pediatr Cardiol Soc 2005;9:371-8.

17. Yu JJ, Kwak BO, Jeon YH, Park YM, Lee R, Chung S, et al. Elevation of the index of left ventricular mass during the acute and subacute phase of Kawasaki disease, and its association with indexes of diastolic function. Cardiol Young 2009;19:64-9.

18. Yamamoto K, Burnett JC Jr, Jougasaki M, Nishimura RA, Bailey KR, Saito Y, et al. Superiority of brain natriuretic peptide as a hormonal marker of ventricular systolic and diastolic dysfunction and ventricular hypertrophy. Hypertension 1996;28:988-94. 